\title{
Synthesis of Aminoethylpiperazine/Formaldehyde Modified Lignin Amine Asphalt Emulsifier and Its Investigation by Online FTIR Spectrophotometry
}

\author{
Lai-Shun SHI ${ }^{1, a,}{ }^{*}$, Tian-Yao WANG ${ }^{1,2}$, Meng CHEN ${ }^{1}$, De-Mei $\mathrm{LI}^{1}$, Cun-Fei MA ${ }^{1}$ \\ ${ }^{1}$ School of Chemistry and Chemical Engineering, Shandong University, Jinan 250061, P. R. China \\ ${ }^{2}$ Shandong An-Tai Chemical Pressure Vessel Inspection Center, Jinan 250014, P. R. China \\ aemail: LSHUNSH@sdu.edu.cn
}

${ }^{*}$ Corresponding author

Keywords: Asphalt, Emulsifier, Lignin, Synthesis, Online FTIR.

\begin{abstract}
A new cationic type asphalt emulsifier of aminoethylpiperazine/formaldehyde modified lignin amine was synthesized by the reaction of lignin, $\mathrm{N}$-aminoethylpiperazine, sodium hydroxide and formaldehyde. The synthesis process was detected by online FTIR technique and the intermediate was found. Based upon the experimental information, a reaction mechanism was suggested for the reaction. The asphalt emulsifier has excellent surface activity and satisfactory emulsification effect. The prepared asphalt emulsion showed higher storage stability. The emulsifier belongs to medium-set emulsifier of asphalt. It can be applied to the road pavement construction of tack coat and chip seal.
\end{abstract}

\section{Introduction}

Asphalt emulsion is a homogeneous system containing asphalt, water and emulsifiers. It can be applied to the road construction. Asphalt emulsion has the advantage of stronger adhesive power with mineral aggregate, lower energy consumption, and less pollution to the environment by comparing with hot asphalt [1-3].

Asphalt emulsifier can be divided into anionic, cationic, zwitterionic and non ionic types. Wates and James reported a polybetaines asphalt emulsifier, which provide superior cationic and anionic emulsions, and slow-setting emulsions at low use levels, and the excellent adhesion of the cured asphalt film [4]. W.T. Zdybak reported an oil-in-water anionic bituminous emulsion, wherein an anionic emulsifier was prepared by reacting lignosulfonate with a sulfonyl chloride or bromide of benzene or substituted benzene or an aliphatic hydrocarbon with four or more carbon atoms [5]. P. Schilling reported a high viscosity cationic slow-set and medium-set emulsions [6]. The emulsifying compositions are combinations of fatty amine and/or polyamine, lignin, and nonionic surfactant. A preferred emulsifying composition can be produced by reacting the combinations of fatty amine and/or polyamine, lignin, and nonionic surfactant with formaldehyde. A lignin amine type asphalt emulsifier, which was using the raw materials of soda lignin, secondary amines and inexpensive aminating assistants, was synthesized by $\mathrm{B}$. Wu et al [7]. Dissymmetric Gemini quaternary ammonium salts cationic asphalt emulsifiers with three carbon chains were synthesized using the raw material of $\mathrm{N}, \mathrm{N}$-dodecyl dimethyl tertiary amine, N,N-octadecyl dimethyl tertiary amine, epichlorohydrin and hydrochloric acid [8]. Liu et al studied the preparation and storage stability of asphalt emulsions made from modified lignin cationic asphalt emulsifiers [9].

In previous papers we have synthesized different types of asphalt emulsifiers [10-13]. With the rapid development of road pavement construction and maintenance, it is necessary to develop and present other new kind of medium-set cationic asphalt emulsifier. Therefore, in this paper a new medium-set cationic asphalt emulsifier was synthesized by the reaction of lignin, $\mathrm{N}$-aminoethylpiperazine, sodium hydroxide and formaldehyde. It can be applied to the road pavement construction of tack coat and chip seal. 


\section{Experimental}

Materials. N-aminoethylpiperazine was of industrial grade. Formaldehyde was of analytical grade and obtained from Laiyang Economic Development Zone Fine Chemical Plant, Laiyang, China. Alkali lignin was of industrial grade, and obtained from Shaoxing Guotai Additives Plant, Shaoxing, China. The asphalt used was AH-90, which was provided by Qilu Petrochemical Company, Zibo, China. The mineral aggregate was the mixture of marble stone in different size.

Synthesis. Scheme 1 shows the synthesis route of aminoethylpiperazine/formaldehyde modified lignin amine.

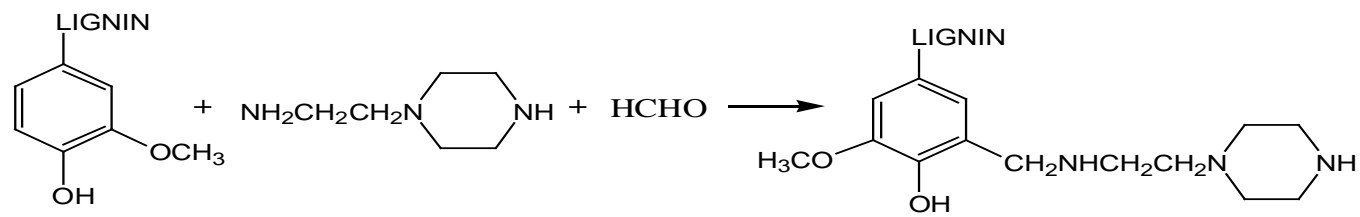

Scheme 1. Synthesis route

$7.0 \mathrm{~g}$ sodium hydroxide and $58.0 \mathrm{~g}$ distilled water was added in a $250 \mathrm{ml}$ three necked flask. Then, $28.3 \mathrm{~g}$ lignin was dissolved, and reacted at $70{ }^{\circ} \mathrm{C}$ for $1 \mathrm{~h} .12 .9 \mathrm{~g} \mathrm{~N}$-aminoethylpiperazine was added. $9.3 \mathrm{~g} 37 \%$ Formaldehyde was added drop wise to the mixed solution. The solution was reacted at $70{ }^{\circ}$ Cfor 5 h.

FTIR Analysis. The FTIR spectra were measured at $400-4000 \mathrm{~cm}^{-1}$ wave number with 32 scans at resolution of $4 \mathrm{~cm}^{-1}$ on a Bruker Tensor-27 FTIR spectrophotometer.

Online FTIR Analysis. The online FTIR detection was operated on a React IR 4000 spectrophotometer (Mettler-Toledo AutoChem, Inc., USA). FTIR spectra were recorded in the range of $650-4000 \mathrm{~cm}^{-1}$ wave number at a resolution of $4 \mathrm{~cm}^{-1} .7 .0 \mathrm{~g}$ sodium hydroxide and $58.0 \mathrm{~g}$ distilled water was added in a $250 \mathrm{ml}$ three necked flask. Then, $28.3 \mathrm{~g}$ lignin was dissolved, and reacted at $70{ }^{\circ} \mathrm{C}$ for $1 \mathrm{~h} .12 .9 \mathrm{~g} \mathrm{~N}$-aminoethylpiperazine was added. The detector of the online FTIR analysis apparatus was immersed in the solution during the reaction process to record the 3D online FTIR spectra. $9.3 \mathrm{~g} \mathrm{37 \%}$ Formaldehyde was added drop wise to the mixed solution in 8-10 min. The solution was reacted at $70^{\circ} \mathrm{C}$ for $5 \mathrm{~h}$. The magnetic stirrer was in use during the online FTIR detection.

Preparation of Asphalt Emulsion. 12.5 g emulsifier was dissolved in $200 \mathrm{~mL}$ water. The pH value of the aqueous solution was adjusted to 2.0 by aqueous $30 \% \mathrm{HCl}$, and the solution was heated to $60-65^{\circ} \mathrm{C} .300 \mathrm{~g} \mathrm{AH}-90$ asphalt was heated to $120-125^{\circ} \mathrm{C}$. The asphalt emulsion was prepared by mixing the emulsifier aqueous solution and asphalt in a RHS-5 type colloid mill for $1 \mathrm{~min}$.

Mixing Experiment. Mineral aggregate (100 g), $1 \mathrm{~g}$ cement and $8 \mathrm{~g}$ water were placed in a 500 $\mathrm{mL}$ bowl. Asphalt emulsion (11 g) was then added. The mixture was mixed and agitated at a speed of 60 revolutions per minute. The mixing time was measured by observation of the mixing performance. The room temperature was $29^{\circ} \mathrm{C}$.

\section{Results and Discussion}

FTIR Characterization. Figure 1 gives the product FTIR spectrum of aminoethylpiperazine /formaldehyde modified lignin amine. In the spectrum, the absorption at $3431 \mathrm{~cm}^{-1}$ (peak 1) is attributed to $\mathrm{O}-\mathrm{H}$ stretching vibration. The absorption at $2923 \mathrm{~cm}^{-1}$ (peak 2) is attributed to $\mathrm{CH}_{2}$ asymmetrical stretching. The absorption at $2810 \mathrm{~cm}^{-1}$ (peak 3) is attributed to $\mathrm{CH}_{2}$ symmetrical stretching. The absorption at $1610 \mathrm{~cm}^{-1}$ (peak 4) is attributed to $\mathrm{N}-\mathrm{H}$ bending vibration. The absorption at $1460 \mathrm{~cm}^{-1}$ (peak 5) is attributed to benzene ring skeletal vibration. The absorption at $1326 \mathrm{~cm}^{-1}$ (peak 6) is attributed to O-H in-plane bending vibration. The absorption at $1222 \mathrm{~cm}^{-1}$ (peak 7) is attributed to Ar-O-C stretching vibration. The absorption at $1126 \mathrm{~cm}^{-1}$ (peak 8) is attributed to 
$\mathrm{C}-\mathrm{H}$ in-plane bending vibration. The absorption at $1000 \mathrm{~cm}^{-1}$ (peak 9) is attributed to C-O and C-N stretching vibration. The absorption at $819 \mathrm{~cm}^{-1}$ (peak 10) is attributed to out-of-plane bending vibration of isolated hydrogen in benzene ring. The absorption at $669 \mathrm{~cm}^{-1}$ (peak 11) is attributed to $\mathrm{O}-\mathrm{H}$ out-of-plane bending vibration. Therefore, the FTIR spectrum agrees with the chemical structure of the product.

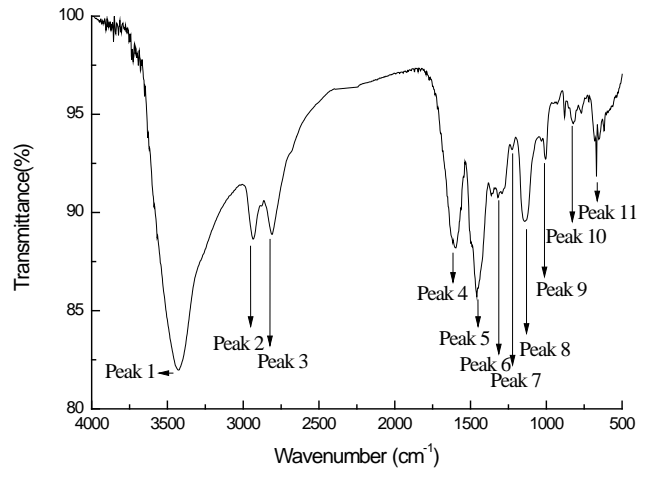

Fig. 1. The FTIR spectrum of the product

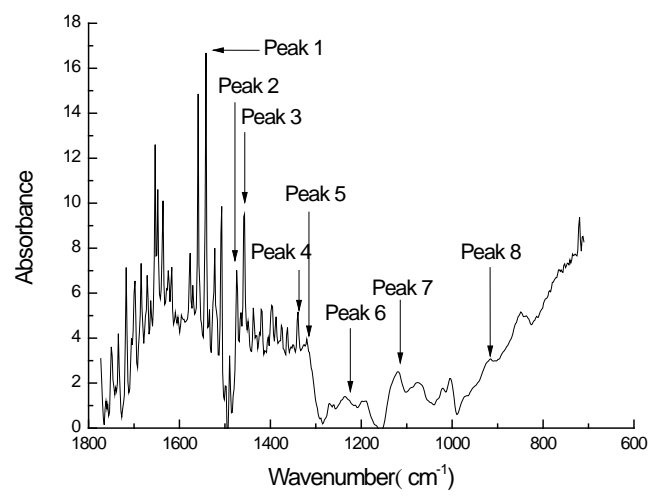

Fig. 3. The FTIR spectrum of component 1

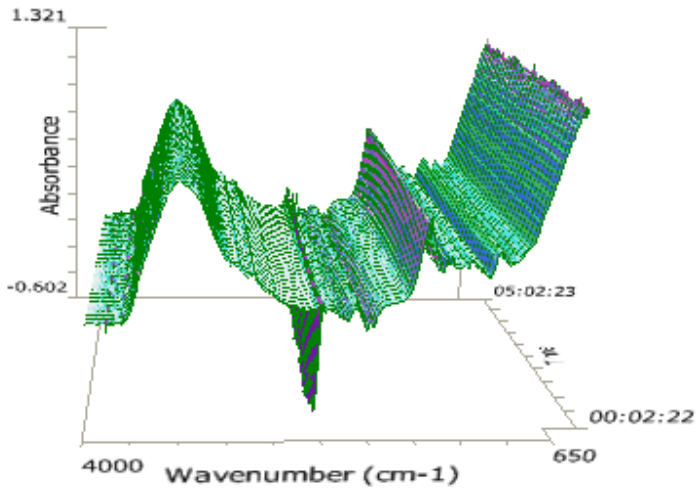

Fig. 2. The 3D online FTIR spectra in the reaction

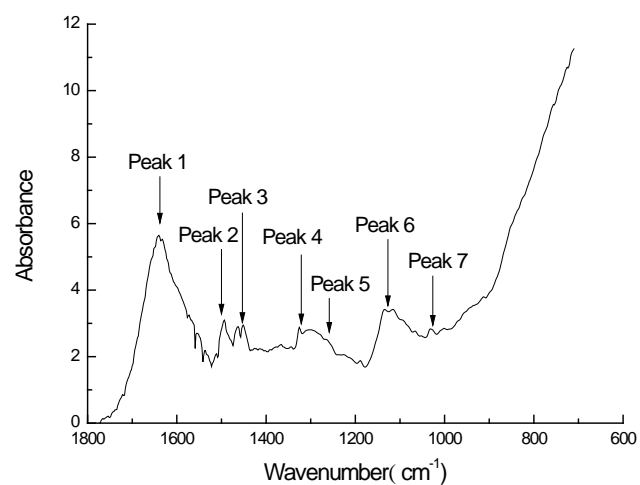

Fig. 4. The FTIR spectrum of component 2

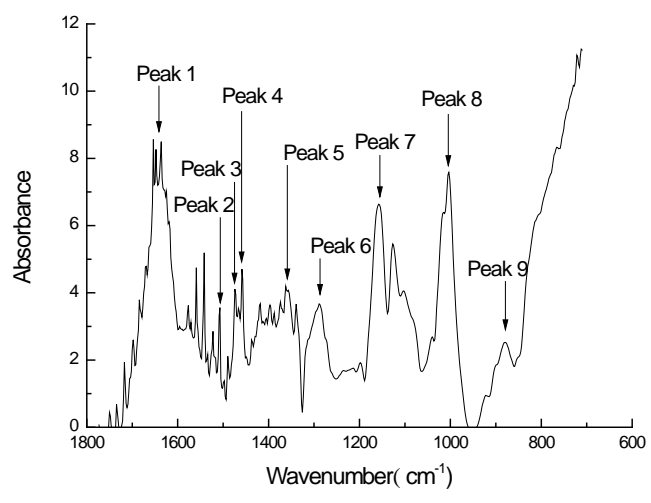

Fig. 5. The FTIR spectrum of component 3

Online FTIR Analysis. The reaction was detected by online FTIR technique. The reaction time was 5.0 h. Figure 2 shows the 3D online infrared spectra in the whole reaction process. As can be seen, the spectra changed a lot.

Three components were found automatically during the online FTIR detection. Its FTIR spectrum was obtained (see Fig. 3-5). In the FTIR spectrum of component 1 (Fig. 3), the absorption at 1542 
$\mathrm{cm}^{-1}$ (peak 1) is attributed to $\mathrm{N}-\mathrm{H}$ bending vibration. The absorptions at $1474 \mathrm{~cm}^{-1}$ (peak 2) and 1456 $\mathrm{cm}^{-1}$ (peak 3) are attributed to methylene scissor vibration. The absorption at $1339 \mathrm{~cm}^{-1}$ (peak 4) is attributed to $\mathrm{O}-\mathrm{H}$ in-plane bending vibration. The absorption at $1320 \mathrm{~cm}^{-1}$ (peak 5) is attributed to methylene out-of-plane swing vibration. The absorption at $1238 \mathrm{~cm}^{-1}$ (peak 6) is attributed to C-N stretching vibration. The absorption at $1122 \mathrm{~cm}^{-1}$ (peak 7) is attributed to C-O-H stretching vibration. The absorption at $922 \mathrm{~cm}^{-1}$ (peak 8) is attributed to O-H out-of-plane bending vibration. Therefore, the spectrum of component 1 obtained by online FTIR should be the intermediate as shown in Scheme 2.

In the FTIR spectrum of component 2 (Fig. 4), the absorptions at $1636 \mathrm{~cm}^{-1}$ (peak 1), $1495 \mathrm{~cm}^{-1}$ (peak 2) and $1449 \mathrm{~cm}^{-1}$ (peak 3) are attributed to benzene ring skeletal vibration. The absorption at $1325 \mathrm{~cm}^{-1}$ (peak 4) is attributed to O-H in-plane bending vibration. The absorption at $1257 \mathrm{~cm}^{-1}$ (peak 5) is attributed to Ar-O-C stretching vibration. The absorption at $1123 \mathrm{~cm}^{-1}$ (peak 6) is attributed to $\mathrm{C}-\mathrm{H}$ in-plane bending vibration. The absorption at $1034 \mathrm{~cm}^{-1}$ (peak 7) is attributed to C-O stretching vibration. Therefore, the FTIR spectrum agrees with the chemical structure of lignin.

In the FTIR spectrum of component 3 (Fig. 5), the absorption at $1636 \mathrm{~cm}^{-1}$ (peak 1) is attributed to $\mathrm{N}-\mathrm{H}$ stretching vibration. The absorptions at $1506 \mathrm{~cm}^{-1}$ (peak 2), $1474 \mathrm{~cm}^{-1}$ (peak 3) and $1458 \mathrm{~cm}^{-1}$ (peak 4) are attributed to benzene ring skeletal vibration. The absorption at $1350 \mathrm{~cm}^{-1}$ (peak 5) is attributed to $\mathrm{O}-\mathrm{H}$ in-plane bending vibration. The absorption at $1290 \mathrm{~cm}^{-1}$ (peak 6) is attributed to Ar-O-C stretching vibration. The absorption at $1152 \mathrm{~cm}^{-1}$ (peak 7) is attributed to $\mathrm{C}-\mathrm{H}$ in-plane bending vibration. The absorption at $1005 \mathrm{~cm}^{-1}$ (peak 8) is attributed to C-O and C-N stretching vibration. The absorption at $877 \mathrm{~cm}^{-1}$ (peak 9) is attributed to out-of-plane bending vibration of isolated hydrogen in benzene ring. Therefore, the FTIR spectrum agrees with the chemical structure of the product, aminoethylpiperazine/formaldehyde modified lignin amine.

Figure 6 gives the plot of relative concentrations $(c)$ of component 1-3 with reaction time. As shown in Fig. 6, the relative concentration of component 1 increases slightly with reaction time. Therefore, we can confirm that component 1 should be the intermediate as shown in Scheme 2 according to the changing trend of concentration and the online FTIR spectrum in Fig. 3.

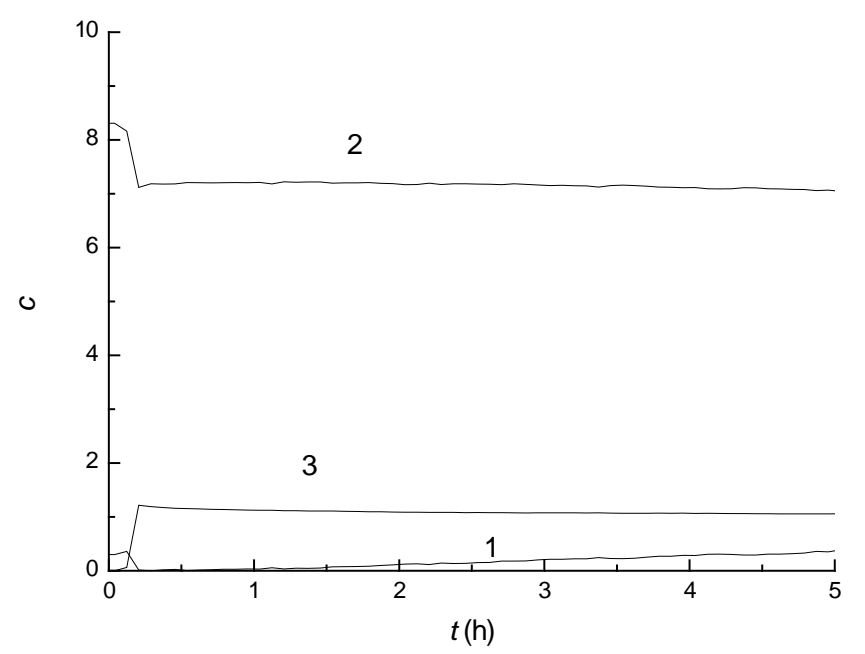

Fig. 6. The relative concentration of component 1-3 versus reaction time

(Curve 1: component 1; Curve 2: component 2; Curve 3: component 3)

The relative concentration of component 2 decreases gradually with reaction time. Therefore, we can confirm that component 2 should be lignin according to the changing trend of concentration and the online FTIR spectrum in Fig. 4.

The relative concentration of component 3 increases with reaction time. Therefore, we can confirm that component 3 should be the target product to be synthesized according to the changing trend of concentration and the online FTIR spectrum as shown in Fig. 5. 
Synthesis Reaction Mechanism. For reaction (R1), we presented a Scheme 2 as shown in reactions (R2-R4), based upon the spectrum obtained by online FTIR detection (Fig. 3-5) and the concentration changing trends of the three components in Fig. 6.

The reaction belongs to a Mannich reaction, which is a third-order reaction based on kinetic study. The $\mathrm{C}_{6}$ position is activated in the lignin structure by the phenolic hydroxyl group. Carbanion is formed by ionization from lignin in alkaline condition (reaction R2). Component 1 of the intermediate is formed by the reaction of $\mathrm{N}$-aminoethylpiperazine with formaldehyde (reaction R3). The final asphalt emulsifier product is formed by the reaction of carbanion with the intermediate (R4), which is a $\mathrm{S}_{\mathrm{N}} 2$ reaction.
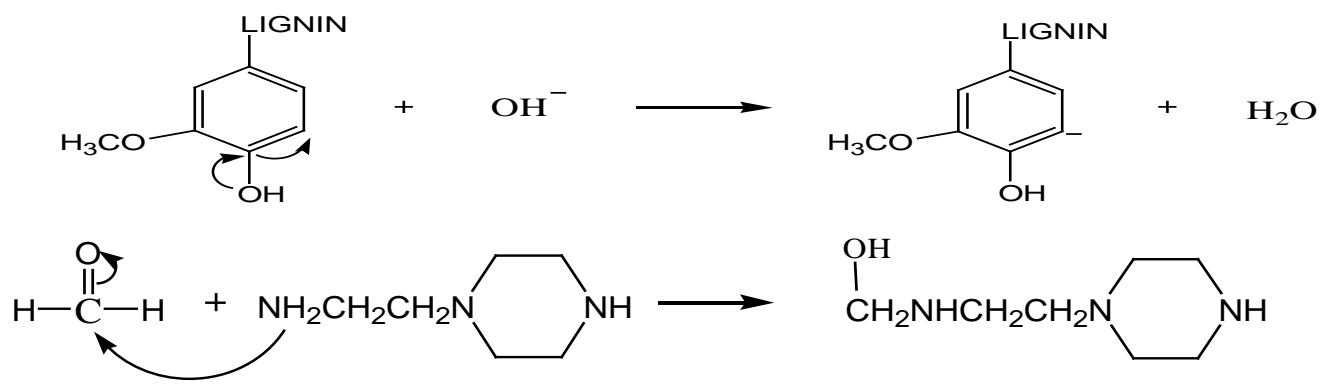

(Component 1: intermediate)

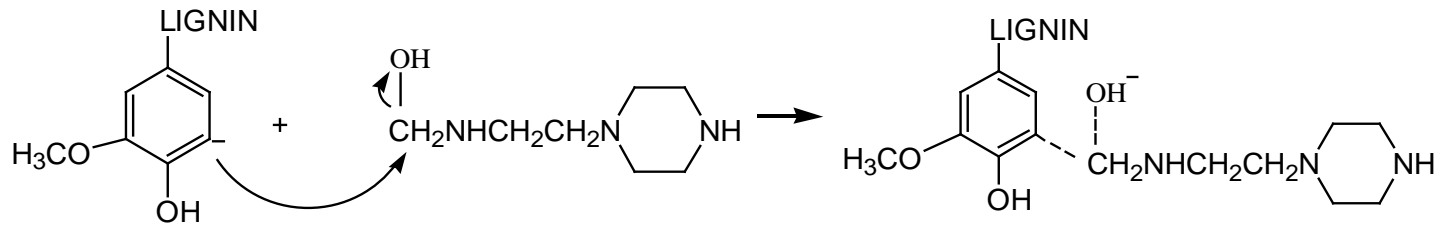

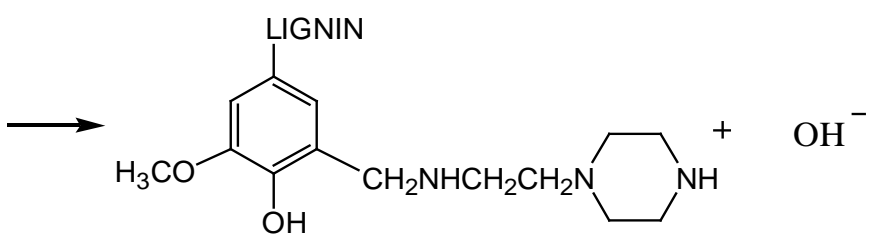

(Component 3: Product)

Scheme 2. The reaction mechanism of synthesis aminoethylpiperazine/formaldehyde modified lignin amine

Performance Evaluation for the Asphalt Emulsion. Emulsifying ability: The emulsifier gives excellent emulsifying ability for AH-90 asphalt. The prepared asphalt emulsion had higher storage stability. There is not asphalt phase and water phase to be separated after 5 days. Mixing performance: The mixing time was $70 \mathrm{~s}$. It indicates that the emulsifier belongs to a medium-set emulsifier of asphalt. It can be applied to the road pavement construction of tack coat and chip seal.

\section{Summary}

A new cationic type asphalt emulsifier was synthesized by the reaction of lignin, $\mathrm{N}$ aminoethylpiperazine, sodium hydroxide and formaldehyde. A reaction mechanism was suggested for the reaction. The emulsifier belongs to medium-set emulsifier of asphalt. It can be applied to the road pavement construction of tack coat and chip seal.

\section{Acknowledgement}

The authors would like to thank the financial support from the Shandong Provincial Science and Technology Project (No. 2015GGX107005). 


\section{References}

[1] H.P. Li, H. Zhao, K. Liao. The preparation of asphalt emulsions with dissymmetric Gemini quaternary ammonium salts cationic surfactants. Energy Sources, Part A, 35 (2013) 2285-2293.

[2] L.E. Chávez-Valencia, E. Alonso, A. Manzano, J. Perez, M.E. Contreras, C. Signoret. Improving the compressive strengths of cold-mix asphalt using asphalt emulsion modified by polyvinyl acetate. Construction \& Building Materials, 21 (2007) 583-589.

[3] G. Barreto, L. Grampre. Bituminous products and aqueous emulsions based on bituminous products and uses thereof. U.S. Patent 7,951,858 B2. (2011)

[4] J.M. Wates, A. James. Polybetaines as asphalt emulsifiers. U.S. Patent 6,540,822 B2. (2003)

[5] W.T. Zdybak. Anionic bituminous emulsions. U.S. Patent 4,427,449. (1984)

[6] P. Schilling. High viscosity cationic slow-set and medium-set emulsions. U.S. Patent 6,077,888. (2000)

[7] B. Wu, Y. Yang, X. Pan, X. Wang, G. Liu. Novel synthetic method of lingninamine-type asphalt emulsifier. J. Northeast For. Univ., 3 (1992) 89-94.

[8] J.F. Lu, H.G. Wang, D. Liu, K.J. Liao. Synthesis of dissymmetric Gemini quaternary ammonium salts cationic asphalt emulsifiers. Industrial Catalysis, 14 (2006) 62-64.(in Chinese)

[9] Z.G. Liu, L. Zong, L.L. Zhao, X.M. Xie. Preparation and storage stability of asphalt emulsions made from modified lignin cationic asphalt emulsifiers. Applied Mechanics and Materials, 357-360 (2013) 781-785.

[10] N. Li, L. Shi, X. Gong, Q. Xu, X. Liu, X. Wang. Synthesis of a novel cationic asphalt emulsifier and its investigation by online FTIR spectrophotometry. Research on Chemical Intermediates, 41 (2015) 1935-1950.

[11] L. Shi, M. Sun, N. Li, B. Zhang. A novel betaine type asphalt emulsifier synthesized and investigated by online FTIR spectrophotometric method. Chem. Ind. Chem. Eng. Q., 21 (2015) $113-121$.

[12] C. Huai, L. Shi, N. Li. Synthesis of a novel betaine-type asphalt emulsifier and its investigation by online FTIR spectrophotometry. Research on Chemical Intermediates, 39 (2013) 597-614.

[13] L. Shi, J. Chen, M. Sun, Y. Wang, S. Lin, Y. Zhang. Synthesis and characterization of a novel cationic type asphalt emulsifier. Applied Mechanics and Materials, 687-691 (2014) 4265-4268. 